zielle Angelegenheit einmischte, dann konnte es sein, dass sowohl der Vater als auch das Familienoberhaupt diesen Ungehorsam mit Schlägen bestraften. Niemand durfte sich den Anweisungen des Familienoberhauptes und seiner Brüder widersetzen. Der Sohn berichtete, auch er sei regelmässig vom Onkel geschlagen worden, wenn er sich getraut habe, etwas gegen die Regeln innerhalb der Familie zu sagen.

\subsection{Wenn die Frau den Mann ver-}

lässt, ist seine Ehre verletzt

Beim zweiten Beschuldigten handelte es sich um einen dreissigjährigen Mann, ebenfalls aus dem Kosovo stammend. Er war verheiratet und hatte zwei Kinder. Dem Beschuldigten wurde vorgeworfen, mehrfach seine Frau mit dem Tode bedroht zu haben, nachdem diese ihn wegen eines anderen Mannes verlassen hatte. Der Beschuldigte bestritt während des Ermittlungsverfahrens sämtliche Vorwürfe, betonte aber immer wieder, dass er wisse, dass seine Frau einen neuen Freund habe, und dass es für diesen Fall nach seinem Brauchtum klare Vorschriften gebe. Es sei an dieser Stelle nochmals auf das
4. Buch, 3. Kapitel, Art. 4 Abs. 3 verwiesen: „Für zwei Dinge hat die Frau die Patrone im Rücken, und für einen Grund darf ihr die Franse geschnitten und sie entlassen werden: für Untreue und für Verletzung der Freundschaft. Für diese beiden Taten der Treulosigkeit tötet der Gatte die Frau; sie bleibt ohne Schutz, ohne Gottesfrieden “. Wird eine Frau untreu, kann der Mann sie töten.

Der Beschuldigte in unserem Fall kannte diese Bestimmung ohne Zweifel. Er betonte jedoch immer wieder, dass er diese Bestimmung nicht vollziehen werde. Er kenne den Kanun zwar, aber er wolle schliesslich nicht, dass seine Kinder ohne Mutter aufwachsen müssten. Aber seine Frau müsse wissen, dass in einem solchen Fall eine „Reaktion" erfolgen könne. Sie habe ihn betrogen, und er könne für nichts garantieren. Seine Brüder seien schliesslich auch noch da, und diese seien ganz besonders auf die Situation in seiner Ehe aufmerksam geworden und könnten eine Trennung unter keinen Umständen dulden, erst recht nicht wenn der Grund dafür die Untreue der Ehefrau sei. Er habe denn seiner Frau auch nie gedroht, sondern sie immer nur auf die Folgen ge- mäss Kanun aufmerksam gemacht und sie darauf hingewiesen, dass sie sich an das alte Brauchtum im Kosovo erinnern solle. Er habe seine Frau daher nicht bedroht, sondern nur "gewarnt“.

Unter anderem wurde dem Beschuldigten auch vorgeworfen, seiner Frau einen Zeitungsartikel geschickt zu haben, in welchem ein Fall eines Ehrenmordes geschildert wurde: Ein Mann hatte seine Exfrau nur wenige Stunden nach der Scheidung niedergestochen.

Barbara Egeler Dormann ist Untersuchungsrichterin in der Schweiz, Rheinstrasse 12, CH-4410 Liestal

\section{Literaturverzeichnis:}

- Ahmeti Zef (2006). Das Strafrecht im Kanun von Lekë Dukagjini. St. Gallen.

- Elsie Robert (2001). Der Kanun - Das albanische Gewohnheitsrecht nach dem so genannten Kanun des Lekë Dukagiini, kodifiziert von Shtjefën Gjeçovi, ins Deutsche übersetzt von Marie Amélie Freiin von Godin. Olzheim/Eifel.

- Fleschenberg Jana (2005). Familiäre Gewalt in Albanien, Hintergrundinformationen. Amnesty International. Aufsatz im Internet.

\title{
Fremdenfeindliche Straftaten und ihre statistische Erfassung - Eine Zwischenbilanz
}

\section{Einleitung}

Kriminalität ist definitionsabhängig und wird unter anderem von den Instanzen sozialer Kontrolle konstruiert. Die Begrifflichkeiten und Definitionen, die der Erfassung und Abbildung krimineller Erscheinungsformen zugrunde gelegt werden, sind ebenso wie die Phänomene selbst zeitlichen Veränderungsprozessen und politischen Strömungen unterworfen. Von offizieller Seite besteht das Bestreben, auf neue Ausprägungen bestimmter Delikte dynamisch zu reagieren. Dies geschieht einerseits durch Akte der Gesetzgebung, was nicht selten mit Strafschärfungen einhergeht. ${ }^{1}$ Zum anderen wird versucht, über Veränderungen der statistischen Erfassung krimineller Verhaltens-weisen eine Anpassung an gewandelte Realitäten vorzunehmen. Letzteres geschah bekanntlich auch in Bezug auf fremdenfeindliche Straftaten. Während diese bis in die 1980er Jahre hinein kaum Beachtung fanden und nicht als besonderes Problem wahrgenommen wurden, gelangten sie im Zuge der Asyldebatte zu Beginn der 1990er Jahre verstärkt ins Blickfeld der Öffentlichkeit. ${ }^{2}$ In dieser Zeit kam es zu zahlreichen Brandanschlägen und Gewaltdelikten (etwa in Solingen, Rostock und Mölln), die großes öffentliches Aufsehen erregten und ferner dazu führten, dass sich die Wissenschaft verstärkt den Hintergründen und Entstehungsbedingungen dieser Taten zuwendete. Kontrollinstanzen, Medien und Politiker sahen sich mit dem Kriminalitätsphänomen „Fremden-feindlichkeit“ konfrontiert, das beobachtet werden musste und deshalb einer statistischen Erfassung bedurfte.

\section{Die Situation bis zum 31.12.2000}

Für die Registrierung von Straftaten der vorgenannten Art stand zunächst die „Po-

\section{Sebastian Presse und Mario Bachmann}

lizeiliche Kriminalstatistik für Staatsschutzdelikte (PKS-S)“ zur Verfügung. Hier erfasste das Bundes-kriminalamt als Teil der Staatsschutzdelikte rechts- und linksextremistische Straftaten. Unter dem zentralen Begriff „Extremismus“ wurden dabei solche Bestrebungen verstanden, die sich gegen den Kernbestand der freiheitlich demokratischen Grundordnung richteten und diese zu überwinden suchten. ${ }^{3}$ Fremdenfeindlichkeit als gesellschaftlicher Hintergrund von Straftaten wurde allerdings nicht eigens ausgewiesen. ${ }^{4}$ Dies geschah erst ab März 1992 in der KPMD-S („Kriminalpoli-zeilicher Meldedienst in Staatsschutzsachen "). Nach der vom Bundeskriminalamt und der von den Länderpolizeien entwickelten Definition handelte es sich bei fremdenfeindlichen Straftaten um solche, die ,in der Zielrichtung gegen Personen begangen werden, denen die Täter aus intoleranter Haltung heraus aufgrund ibrer Nationalität, Ras- 
se, Hautfarbe, Religion, Weltanschauung, Herkunft oder auf Grund ihres äußeren Erscheinungsbildes ein Bleibe- oder Aufentbaltsrecht in ibrer Wobnumgebung oder in Deutschland bestreiten ". 5 Als kriminalpolitische Folge der Asyldebatte und der Brandanschläge wurde Fremdenfeindlichkeit seitens der Sicherheitsbehörden gewisser-maßen als Ausdruck „privater Abschiebung“ verstanden und die diskriminierende Tirade „Ausländer Raus!“ mittels vorgenannter Definition operationalisiert. ${ }^{6}$

Blickt man auf die statistischen Befunde der 1990er Jahre, ist festzustellen, dass nach der Hochphase fremdenfeindlicher Straftaten in den Jahren 1992 und 1993 mit jeweils über 6.000 Fällen, ein deutlicher Rückgang auf etwa 2.000 bis 3.000 Taten pro Jahr stattgefunden hat. ${ }^{7}$ Auffällig ist diesbezüglich sowohl die Dominanz der Propagandadelikte (rund 70\%) als auch der vergleichsweise geringe Anteil von Gewaltdelikten (etwa 8 bis 11 versuchte Tötungen sowie 300 bis 400 Körperverletzungen jährlich). ${ }^{8}$

\section{Das Erfassungssystem „Politisch motivierte Kriminalität“ (PMK) seit 2001}

\section{Gründe für die Änderung der Registrierung}

Die erläuterte Definitionssystematik wurde in vielerlei Hinsicht für unzureichend erachtet, da ihr Mängel auf verschiedenen Ebenen zu Grunde lagen. Im Wesentlichen kann man von ungenügen-dem Begriffsdesign (Kreationsebene), problematischer Fallzuordnung (Ermittlungsebene) und unzureichender Vergleichbarkeit (Darstellungsebene) sprechen, wobei diese Kategorien nicht trenn-scharf voneinander abzugrenzen sind und die Defizite teils in wechselseitiger Beziehung stehen.

\section{a) Kreationsebene}

Zunächst stellte sich die Frage, ob mit den restriktiven Maßstäben zur systemüberwindenden oder bleiberechtsverweigernden Motivation des Täters die richtigen Erfassungskriterien zu Grunde gelegt worden waren. Untersuchungen ergaben, dass das Problemfeld „Straftaten gegenüber Migranten " keinesfalls nur von diesen Beweggründen motiviert sein musste. ${ }^{9}$ Der ideologisch motivierte, rechtsextremistische Täter, der über ein verfestigtes Weltbild verfügt, stellt in der Wirklichkeit eher eine Ausnahme dar. ${ }^{10}$ Weitaus typischer ist der „anpolitisierte“ Mitläufer ${ }^{11}$, den eine starke Gruppenorientierung auszeichnet und bei dem ausländerfeindliche Gesinnungen, Gewaltbereitschaft und rechtsextremistische Ideologien wenig ausgeprägt sind. Vor dem Hinter-grund, dass es sich bei der Mehrzahl der Tatverdächtigen um Jugendliche und junge Erwachsene handelt, ${ }^{12}$ liegen oftmals jugendtypische Beweggründe für die Tatbegehung wesentlich näher. ${ }^{13}$

\section{b) Ermittlungsebene}

Auf polizeilicher Ebene wirkte sich für die ermittelnden Beamten erschwerend aus, dass die erwähnte Definition der Fremdenfeindlichkeit eine stark tatmotivationsabhängige Komponente aufwies, die sich im polizeilichen Ermittlungsverfahren nur selten ohne Weiteres feststellen ließ. ${ }^{14}$ Insoweit bestand die Gefahr der generellen Unterstellung einer politischen Tatmotivation und der daraus resultierenden Einordnung einer Tat etwa als „fremdenfeindlich “. ${ }^{15}$ Andererseits kam es möglicherweise zur statistischen Nichterfassung, wenn Motive geleugnet bzw. gar nicht erst bekannt wurden oder bei Nichtvorliegen von Hinweisen pauschal eine fehlende Tätermotivation angenommen wurde. ${ }^{16}$ Objektivierungsversuche hinsichtlich politischer Motive scheiterten daran, dass die Täter bei einer Vielzahl von Delikten nicht zu ermitteln waren. ${ }^{17}$ Vor diesem Hintergrund verwundert es nicht, dass weder auf Ebene der Länder, noch auf derjenigen der Präsidien, Direktionen und Inspektionen ein einheitlicher Umgang mit den Begriffen festgestellt werden konnte. ${ }^{18} \mathrm{Ob}$ eine Tat als fremdenfeindlich charakterisiert wurde, hing vom Problembewusstsein und dem subjektiven Ermessen der ermittelnden Beamten ab. Aufgrund von Vorannahmen und lokal unterschiedlichen Abgrenzungskriterien wurden manche Fälle ausgefiltert und nicht erfasst, wie z.B. soziale Nahkonflikte oder Nachbarschaftsstreitigkeiten. Propagandadelikte, die man vorher nicht erfasst hatte, wurden nach spektakulären Ereignissen und in Zeiten hoher Arbeitsbelastung eher als fremdenfeindlich bewertet und erfasst. ${ }^{19}$ Andererseits kam es auch vor, dass manche Delikte aus „Opportunitätsüberlegungen“ - um einem schlechten Image des eigenen Bundeslandes oder Dienstbezirkes vorzubeugen - nicht unter die entsprechenden Definitionen subsumiert wurden. ${ }^{20}$

\section{c) Darstellungsebene}

Als dritter Schwachpunkt erwies sich schließlich die Tatsache, dass durch die unzuverlässigen Eingaben aus verschiedenen Institutionen mehrere Statistiken mit Zahlen ganz unterschiedlicher Delikte versorgt worden sind. ${ }^{21}$ Sowohl leichte als auch schwerste Straftaten wie Brandstiftungs- und Tötungsdelikte gelangten unter dem Stichwort „fremdenfeindlich“ in die Statistiken. Das Nebeneinander verschiedenster Meldedienste verhinderte zudem eine gute Vergleichbarkeit. So wurden Delikte, die den Bereich des polizeilichen Staatsschutzes betrafen, nicht selten mehrfach erfasst: in der Polizeilichen Kriminalstatistik (PKS), der PKS-S sowie im KPMD-S. Während die beiden erstgenannten als Ausgangsstatistiken geführt wurden, beruhen die Zahlen der KPMD-S auf einer Eingangsmeldung. Die Meldedienstzahlen lagen in den Jahren 1997 bis 1999 bei rechts-extremistischen Straftaten zwischen 20 und $40 \%$ über den Daten der PKS-S, bei linksextremen Delikten sogar zwischen 40 und $110 \%{ }^{22}$ Aufgrund der vorgegebenen Erfassungsmöglichkeiten in den Statistikbögen der PKS-S war es kaum möglich, eine Straftat entsprechend der aktuellen Phänomenologie der Staatsschutzkriminalität statistisch abzubilden, weil Fremdenfeindlichkeit - wie bereits erwähnt - dort nicht eigens ausgewiesen war. ${ }^{23}$

\section{Die Zuordnungskriterien seit 2001}

Seit dem 1.1.2001 versuchen Bund und Länder nunmehr, politisch motivierte Kriminalität mit anderen Kriterien zu erfassen. Der erfasste Sachverhalt wird dabei im Rahmen einer mehr-dimensionalen Betrachtung unter verschiedenen Gesichtspunkten bewertet. Dies bedeutet konkret, dass die Ermittlungsbehörde Feststellungen zum subjektiven Tathintergrund, zur Deliktsqualität, zur objektiv thematischen Zuordnung der Tat sowie zu deren gegebenenfalls einschlägigen internationalen Dimension oder extremistischen Ausprägung trifft. ${ }^{24}$ Eine politisch motivierte Straftat ist u.a. dann gegeben, wenn ein Fall der „Hasskriminalität“ vorliegt. Hierfür ist entscheidend, ob „die Umstände der Tat oder die Einstellung des Täters darauf schließen lassen, dass sie sich gegen eine Person aufgrund ibrer politischen Einstellung, Nationalität, Volkszugehörigkeit, Rasse, Hautfarbe, Religion, Weltanschauung, Herkunft, sexuellen Orientierung, Behinderung oder ibres äußeren Erscheinungsbildes bzw. ihres gesellschaftlichen Status 
richtet. “25 Das Themenfeld „Hasskriminalität" besteht wiederum aus verschiedenen Untergruppen, zu denen auch diejenige der „fremdenfeindlichen Straftat“ gehört. Unter einer solchen wird nun - anders als bis Dezember 2000 - der Teil der Hasskriminalität verstanden, der aufgrund der tatsächlichen oder vermeintlichen Nationalität, Volkszugehörigkeit, Rasse, Hautfarbe, Religion, Herkunft des Opfers verübt wird. "26 Insofern ist es also zu einer Erweiterung des Begriffsverständnisses gekommen, da es nicht mehr nur um die Bleiberechtsverweigerung gehen soll. Schließlich ist darauf hinzuweisen, dass die Staatsschutzdelikte des StGB, zu denen insbesondere die Propagandastraftaten nach $\mathbb{S} \int 86$, 86a gehören, generell als politisch motivierte Kriminalität registriert werden, und zwar auch dann, wenn im Einzelfall keine politische Motivation festgestellt werden kann. ${ }^{27}$

\section{Statistische Befunde}

Blickt man auf die statistischen Daten seit Änderung des Erfassungssystems, fällt Folgendes auf: Während sich die Zahl der politisch motivierten Straftaten mit fremdenfeindlichem Hintergrund bis 2005 auf etwa 2.500 Fälle jährlich einpendelte, kam es im Jahr 2006 zu einem deutlichen Anstieg (3.294 Fälle). In den folgenden Jahren fand jedoch ein Rückgang auf das Ausgangsniveau des Zeitraumes bis 2005 statt (2007: 2.866; 2008: 2.950; 2009: 2.477). ${ }^{28}$ Bei den fremdenfeindlichen Gewalttaten ergibt sich ein ähnliches Bild. Nach einer Abnahme bis 2005 auf 373 Taten, ist für 2006 eine beachtliche Zunahme auf 511 Delikte zu verzeichnen. Seitdem sind die Zahlen kontinuierlich rückläufig (2007: 440; 2008: 409; 2009: 366). Die auffällig hohen Werte 2006 dürften u.a. auf die in diesem Jahr infolge von herausgehobenen Straftaten und anlässlich der Fußballweltmeisterschaft in den Medien intensiv geführte Diskussion über fremdenfeindliche Kriminalität zurückzuführen sein (Nachahmungstaten; verstärkte polizeiliche Präsenz während der Fußballweltmeisterschaft und dadurch erhöhte Kontrolldichte; Sensibilisierung der Bevölkerung mit einer sich hieraus ergebenden erhöhten Anzeigebereitschaft). ${ }^{29}$

\section{Akzeptanz der PMK}

Als positive Folge der PMK wird - zumeist von behördlicher Seite - auf dessen Erfassungsweite abgehoben. Das System von Begrifflichkeiten bilde das wahrgenommene Aufgabengebiet realistisch und umfassend ab. Dabei könnten auch Einzelphänomene in ihrer Gesamtheit erfasst werden, ohne jedes Delikt und jeden Täter mit dem unterstellten Motiv der Systemüberwindung belegen zu müssen. Dadurch ergäben sich Chancen einer differenzierten Auswertung und Lagedar-stellung politisch motivierter Kriminalität, die Grundlage für effiziente präventive und repressive Maßnahmen seien. ${ }^{30}$ Das System dokumentiere das tatsächliche Ausmaß politisch motivierter, insbesondere rechtsextremistischer Gewalt besser als das vorherige und ermögliche es, auf systematischer Basis ein vollständiges Bild über deren Ausmaß und Opfer zu zeichnen. Dies sei nicht zuletzt für die Konzeption von Gegenmaßnahmen bedeutsam und leiste einen wichtigen Beitrag zu mehr Rechtssicherheit in Anwendung und Umsetzung in der Praxis. ${ }^{31}$ Die Straftaten könnten nach Auskunft des BKA aufgrund der Mehrdimensionalität des Systems besser zugeordnet werden, die Straftat werde differenzierter dargestellt als im alten System. ${ }^{32}$ Die Feststellung der persönlichen Beweggründe anhand der Definition von Hasskriminalität werde den Behörden erleichtert, da die nunmehr vorzunehmende Gesamtwürdigung der Tat Rückschlüsse auf politische Motivationen gestatte. Der Terminus Hasskriminalität lehne sich zwar an den amerikanischen Begriff „hate crimes“ an, doch unterscheide er sich durch umfangreichere Umschreibung vom US-Vorbild. Mit der Erfassung des gesellschaftlichen Status erreiche der deutsche Ansatz im Vergleich zum amerikanischen eine Erweiterung des ursprünglichen Verständnisses. ${ }^{33}$

Auch aus Sicht der verantwortlichen Bundesministerien ist die Einführung des neuen Definitions-systems ein Erfolg. Schon im Ersten Periodischen Sicherheitsbericht wurde die Erwartung geäußert, dass die Neuregelung Unsicherheiten und Inkonsistenzen in erheblichem Maße beseitigen werde ${ }^{34}$ und präzisere Definitionen und verfahrensrechtliche Regelungen die Arbeit von Polizeibeamten handhabbarer machen und möglicherweise auch erleichtern werde ${ }^{35}$. Bereits im Zweiten Periodischen Sicherheitsbericht folgte dann die entsprechende Bestätigung, indem dort festgestellt wird, dass die Polizei die Umstellung insgesamt als erfolgreich bewerte. ${ }^{36}$ Diese Einschätzung sagt aber allenfalls etwas über die verwaltungstechnische Brauchbarkeit des neuen Erfassungssystems aus, nichts jedoch über die statistische Güte der erfassten Ergebnisse. Im Folgenden soll daher ein etwas kritischerer Blick auf das Erfassungssystem PMK geworfen werden.

\section{Kritik und Zweifel am Registrierungssystem PMK}

\section{a) Subjektive Einflüsse im Erfassungsprozess}

Hinsichtlich der Klassifizierung einer Tat als politisch motivierte Kriminalität, die in einem sehr frühen Stadium des Ermittlungsverfahrens erwartet und getroffen wird, bleibt ein beachtlicher subjektiver Faktor erhalten. ${ }^{37}$ Insoweit bestehen vor allem deshalb Bedenken, weil der polizeiliche Sachbearbeiter die - im Vergleich zum tatbestandsbezogenen Vorsatz - schwierige Aussage zur Motivation des Täters treffen hat. ${ }^{38}$ Lässt sich dieser nicht entsprechend ein, kann sich eine Beurteilung nur auf die äußeren Umstände der Tat stützen. ${ }^{39}$ Im Gegensatz zu Propaganda- oder Agitationsdelikten, denen man eine Aussage leichter nahe legen kann, ${ }^{40}$ lassen sich aus äußeren Bedingungen nicht ohne weiteres Rückschlüsse auf die Tätermotivation ableiten, da es insbesondere bei Gewaltdelikten häufig an einer Aussage fehlt. ${ }^{41}$ Bezüglich der Erfassung und Weitergabe rechts motivierter Gewalttaten ist die Befürchtung von interessengeleiteten Selektionsprozessen und Manipulationen durch bestimmte Bundesländer nicht ganz fern liegend; wer sich um den guten Ruf als Wirtschafts- oder Tourismusstandort bemüht, tut gut daran, den Eindruck von Fremdenfeind-lichkeit zu vermeiden. ${ }^{42}$ Angesichts der Tatsache, dass etwa 70 Prozent der jungen fremdenfeindlich handelnden Täter im Alter von unter 21 Jahren sind ${ }^{43}$ und nur selten aus politischen Beweggründen handeln, kann die Frage aufgeworfen werden, ob hier überhaupt von politischen Delikten gesprochen werden kann. Denn es erscheint äußerst zweifelhaft, ob der fragliche Täterkreis ein politisches Bewusstsein ausgebildet hat, das seine Gewalthandlungen steuert. ${ }^{44}$ Darüber hinaus ist fraglich, wie im Falle einer Kumulation oder Überlagerung verschiedener Tatmotive zu verfahren ist. Solche Bewertungsprobleme ergeben sich im Bereich fremdenfeindlicher Straftaten u.a. dann, wenn der Täterwunsch nach Selbstdarstellung in der Gruppe ebenso wichtig erscheint wie die Ablehnung einer Fremdgruppe. ${ }^{45}$ Ebenfalls schwer zu verifizieren ist die Selbstdeklaration ${ }^{46}$ einer Tat als „politisch“, die etwa aus Gründen des Geltungsbedürfnisses randständiger, sozial benach-teiligter Täter erfolgt. Die Zuordnung von Taten in ein „politisches“ Erfassungssystem ist daher insgesamt problematisch. 


\section{b) Importierter Modellbegriff}

Das Modell der Hassverbrechen (, ,hate crimes“), das dem Registrierungssystem PMK zu Grunde liegt, stammt aus den USA und hatte vor dem Hintergrund tiefgreifender kultureller und ethnischer Segregation in erster Linie sozialemanzipatorische und repressive Zielsetzungen. Dort wurden Hasstaten zum Thema gemacht, um benachteiligte Gruppen zu stärken. ${ }^{47}$ In diesem Zusammenhang wurde der Skandalbegriff genutzt, um Diskriminierung und Gewalttätigkeiten gegen bestimmte gesellschaftliche Gruppen öffentlich anzuklagen - Kriminalpolitik wurde zu einem Vehikel der Identitätspolitik selbstbewusster Minderheiten. ${ }^{48}$ Belastungen zwischen verschiedenen ethnischen und religiösen Bevölkerungsteilen sowie Gruppenkonflikte eines traditionellen Einwanderungs-landes wie den USA sind mit denen Deutschlands kaum zu vergleichen. Hierzulande kann den Verfechtern einer unkritischen Begriffsübernahme (mit dem Verweis auf eine positivere soziale Ausgangslage) der Gedanke wirksamer Integrationsmaßnahmen entgegengehalten werden, die ein weit geringeres Potential haben, durch öffentliche Stellungnahmen zu Nachahmungseffekten und einer Konflikteskalation zu führen. ${ }^{49}$ In dem seit einigen Jahren andauernden Diskurs einer breiteren Öffentlichkeit in Deutschland über "hate crimes“ dominiert jedoch ein positives Vorurteil gegenüber diesem Konzept bzw. den damit verbundenen politischen Forderungen. ${ }^{50}$ So können „hate crimes“ leicht für eine objektive Kategorie gehalten werden, in der sich die Realität eines vermeintlich neuen Typs von Kriminalität spiegele. ${ }^{51}$ Problematisch erscheint der Prozess der Etablierung von „hate crime" oder den deutschen Bezeichnungen „Hassverbrechen“ bzw. „Hasskriminalität" als neuen Fahnenwörtern, wozu auch die Implementierung von Neologismen wie „Hassgruppen“, „-botschaften“ oder „-aktivisten“ gehört. ${ }^{52}$ Diese Fahnen werden gewöhnlich von Interessensvertretern mit der Absicht aufgezogen, ihre eigene Problemdefinition gesellschaft-lich zu etablieren. ${ }^{53}$ Erfolgreich durchgesetzte (Gewalt),Feststellungen“ ziehen sodann regelmäßig repressive Konsequenzen nach sich. ${ }^{54} \mathrm{Ob}$ die statistischen Befunde, die mittels der Einführung des Begriffes „Hasskriminalität“ erhoben werden, der Kriminalpolitik Ansätze liefern, die über den engen Horizont des Strafrechts hinausgehen, ist schon deshalb fraglich, weil der Nutzen dieser Kategorie - auch angesichts empirischer Defizite - nicht geklärt ist. ${ }^{55}$ Kriminalstatistische
Daten-sammlungen sind keine objektiven Messfühler der Kriminalitätswirklichkeit, ${ }^{56}$ sondern behördliche Arbeitsnachweise und kriminalpolitische Werkzeuge. So müssen bei ihrer Interpretation die jeweiligen Dokumentations- und Implementationsinteressen bestimmter Gruppen in die Betrachtung mit einbezogen werden. ${ }^{57}$ Insofern ist auch der stärker ausdifferenzierte deutsche Ansatz, der mit dem Erfassungskriterium des gesellschaftlichen Status eine Erweiterung des ursprünglichen Verständnisses im Sinne eines potentiell expansiven Konzeptes herbeiführt ${ }^{58}$ und einen großen Bereich von Tätern und Situationen abdeckt, kritisch zu beurteilen. Denn ein Katalog, der jederzeit geöffnet und ergänzt werden kann, bietet keine Handlungs- und Erfassungssicherheit, sondern führt zu Beliebigkeit und Unschärfe bei der Einordnung sozialer Konflikttatbestände. ${ }^{59}$ So werden Gewalttätigkeiten, die im Rahmen bestimmter Sportereignisse gegen rivalisierende Gruppen und Unbeteiligte ausgeübt werden, in Spanien bereits als Hasskriminalität erfasst. ${ }^{60}$

\section{c) Gruppenfokussierung}

Die Gruppenfokussierung des aktuellen Erfassungssystems sorgt dafür, dass andere Beweggründe einer Tat zugunsten des Motivs der Gruppenfeindschaft vernachlässigt werden. Das häufig komplexe Geschehen wird reduziert und auf den Aspekt der Gruppenfeindschaft hin vereinfacht. ${ }^{61}$ Das Opfer tritt hinter den öffentlichen Sicherheits- und Friedensbotschaften zurück. Fraglich ist zudem, inwiefern der gruppenhass- oder vorurteilsbedingte Sondercharakter einer Tat Einfluss auf ihre Gewichtung haben darf, da durch eine Bedeutungssteigerung längerfristige Strafschärfungs-tendenzen zu erwarten sind. ${ }^{62}$ Ein Verbrechen, das aus Gruppenfeindschaft begangen wurde, soll in den Augen mancher ein stärkeres Gewicht bekommen als eine vergleichbare Tat, bei der Vorurteile keine Rolle spielen. So seien die erstgenannten Taten umfassender angelegt, zielten über das einzelne Opfer hinaus auf dessen Gruppe und stellten auch einen Angriff auf die gesellschaftliche Vielfalt als solche dar. Nicht zuletzt seien sie für das Opfer schwerer zu verarbeiten. ${ }^{63}$ Das letztgenannte (empirische) Argument, lässt sich nicht überprüfen, solange nicht einschlägige Studien vorliegen, die systematisch mit Vergleichsgruppen arbeiten ${ }^{64}$. Plausibler ist ohnehin die Gegenthese, die auf eine CopingStrategie hinweist, die sich aus der Gruppenzugehörigkeit als solcher erst ergeben kann: Gerade wenn sich der Geschädigte einer selbstbewussten Minderheit zugehörig fühlt, ist er in der Lage, seine Selbstachtung zu erhalten, indem er die Verantwortung für die Tat vollständig den Tätern und Ihren Vorurteilen zuschreibt. ${ }^{65}$

\section{d) Indikatorwirkung und „Trigger"- Funktion leichter Straftaten}

Obwohl der Nachweis einer unmittelbaren „Trigger"- Funktion für gravierendere Folgetaten fehlt, sind in der Perspektive der aktuellen Kriminalpolitik leichte Straftaten wie Propagandadelikte Ausgangspunkt und Ausdruck erheblicher Sozialgefahren. ${ }^{66}$ Durch ihre zwingende Registrierung als politisch motivierte Kriminalität werden sie in einen Zusammenhang gestellt, der den Eindruck erweckt, als handele es sich stets um besonders gefährliche Rechtsbrüche. Es ist aber keineswegs bewiesen, dass Propagandadelikte häufig schlimmeren Straftaten vorausgehen. ${ }^{67}$ Zudem fehlt bislang der Nachweis für die gelegentlich anzutreffende These, dass es sich bei Taten nach $\$ \mathbb{S} 86,86 a$ $\mathrm{StGB}$ um Indikatoren für die Vorurteilsverbreitung innerhalb einer Gesellschaft handele, die den Zustand der Beziehungen zwischen verschiedenen Bevölkerungsgruppen wie eine Art Messinstrument abbildeten. ${ }^{68}$ Die Terminologie von „alarmierenden Befunden “ und Forderungen nach einem „Wehret den Anfängen “69, erscheinen zwar griffig und populär, beruhen aber auf der Annahme von Zusammenhängen, die unbewiesen sind. Wenn klangvolle Bruchstücke komplexe Erklärungen ersetzen, kann allein durch die Begriffssymbolik, das Abzielen auf Vorstellungswelten von Kriminalität und einer Emotionalisierung, die auf das Sicherheitsgefühl Einfluss hat, einer größeren Verbrechensfurcht Vorschub geleistet werden; längerfristig ist die Konstruktion sozialer Konfliktmuster und neuer Problemperspektiven zu befürchten. ${ }^{70}$

\section{Fazit}

Nach inzwischen fast zehn Jahren bleibt festzuhalten, dass auch das aktuelle Erfassungssystem der PMK defizitär ist und unter ähnlichen Mängeln leidet wie sein Vorgänger. Es drängt sich deshalb die grundsätzliche Frage auf, ob vielschichtige Phänomene wie die Beeinträchtigung von Migranten durch deutsche Straftäter überhaupt zum Gegenstand von Erfassungsversuchen gemacht werden können. Angesichts der Komplexität derartiger Erscheinungsformen von Kriminalität muss bezweifelt werden, dass sie mit engen 
Definitionen registrier- und darstellbar sind. Die durch Begriffe wie „Hasskriminalität“ oder „Fremdenfeindlichkeit" nahe gelegten monokausalen Ursachen gibt es in aller Regel nicht. ${ }^{71}$ Kategorien wie ,fremdenfeindlich“ oder „rechtsextrem“ sind Konstrukte und nicht zuletzt das Ergebnis von Definitions- und Zuschreibungsprozessen, die in der Vergangenheit oftmals beliebig und zufällig geschehen sind. ${ }^{72}$ Die auf dieser Grundlage ermittelten Daten sollten deshalb nicht überbewertet werden. Vor allem besteht für Gesetzesinitiativen wie diejenige der Länder Mecklenburg-Vorpommern und Sachsen-Anhalt, die u.a. das Ziel verfolgen, fremdenfeind-liche Tatmotive durch Änderung der $\int \mathbb{S} 46,47$ und 56 StGB strafschärfend zu berücksichtigen, keine Notwendigkeit. ${ }^{73}$ Erforderlich ist es hingegen über Alternativen oder wenigstens Verbes-serungen zum derzeitigen Erfassungssystem PMK nachzudenken.

Der Autor Presse ist stud. Hilfskraft am Institut für Kriminologie der Universität zu Köln bei Professor Dr. Frank Neubacher M.A. - spresse@uni-koeln.de; der Autor Bachmann ist ebendort als wiss. Mitarbeiter und Doktorand tätig - Mario.Bachmann@ uni-koeln.de

\section{Fußnoten:}

1 Vgl. jüngst etwa den Gesetzesantrag der Länder Mecklenburg-Vorpommern und SachsenAnhalt vom 10.02.2010 (BR-Drs. 71/10), der eine Änderung der $\mathbb{S} \mathbb{S} 46,47$ sowie 56 StGB vorsieht und vor allem fremdenfeindliche Tatmotive stärker strafschärfend berücksichtigen will; vgl. dazu auch V.

2 Vgl. Neubacher: Fremdenfeindliche Brandanschläge, 1998, S. $1 \mathrm{ff}$.

3 Vgl. Kubink: Fremdenfeindliche Straftaten - ein neuer Versuch der polizeilichen Registrierung und kriminalpolitischen Problembewältigung, in: MSchrKrim 2002, 325 (326). Auch die Propagandadelikte $(\mathbb{S} 86,86$ a $S t G B)$ wurden unter dieser Definition erfasst.

$4 \mathrm{Vgl}$. BMI/BMJ: 1. Periodischer Sicherheitsbericht, 2001, S. 266.

5 Vgl. Kubink: Rechtsextremistische und fremdenfeindliche Straftaten, in: ZRP 2002, 308 (308).

6 Vgl. Schüler-Springorum: Fremdenfeindliche Straftaten (Buchbesprechung), in: MSchrKrim 1999, 142 (143).

7 Vgl. BMI/BMJ (Anm. 4), S. 285; im Jahr 2000 wurden allerdings 3.591 Fälle registriert.

8 Näher hierzu BMI/BMJ (Anm. 4), S. 286.

9 Vgl. Peucker/Gaßebner/Wabl: Analyse polizeilicher Ermittlungsakten zu fremdenfeindlichen, antisemitischen und rechtsextremistischen Straftaten, in: Wahl (Hg.): Fremdenfeindlichkeit, Antisemitismus, Rechtsextremismus, 2001, S. 12 ff.; Kubink: Fremdenfeindliche Straftaten, 1997, S. 139, 195 ff.; Willems: Fremdenfeindliche Gewalt, 1993, S. 246.

10 Vgl. Kubink (Anm. 9), S. 139.

11 Vgl. Willems (Anm. 9), S. 95.

12 Vgl. Peucker/Gaßebner/Wahl (Anm. 9), S. 26.

13 Vgl. Kubink (Anm. 9), S. 139.
14 Vgl. Eisenberg: Kriminologie, 6. Aufl. 2005, \ 45 Rn. 147 f.; Schneider: Politische Kriminalität: Hassverbrechen, in: Kriminalistik 2001, 21 (22).

15 Vgl. Eisenberg (Anm. 15), \$ 45 Rn. 147.

16 Vgl. BMI/BMJ (Anm. 4), S. 270.

17 Vgl. Reichard: Die Behandlung fremdenfeindlicher Straftaten im deutschen Strafrecht, 2009, S. 17.

18 Vgl. Singer: Erfassung der politisch motivierten Kriminalität, in: Kriminalistik 2004, 32 (33).

19 Vgl. Kubink (Anm. 9), S. 246.

20 Vgl. Holzberger: Offenbarungseid der Polizeistatistiker. Registrierung rechtsextremistischer Straftaten, in: Bürgerrechte \& Polizei, Cilip 2001, S. 26 (29); Singer (Anm. 18), 32 (36).

21 Vgl. Peucker/Gaßebner/Wabl (Anm. 9), S. $19 \mathrm{ff}$.

$22 \mathrm{Vgl} . B M I / B M J$ (Anm. 4), S. 271.

23 Vgl. dazu auch Singer (Anm. 18), 32 (33).

$24 \mathrm{Vgl}$. BMI/BMJ: 2. Periodischer Sicherheitsbericht, 2006, S. 135 .

$25 \mathrm{Vgl}$. BMI/BMJ (Anm. 24), S. 135

26 Vgl. Reichard (Anm. 17), S. 8.

27 Vgl. Keiser: Unerlässliches zur Verteidigung der Rechtsordnung gegen sogenannte Hasskriminalität, in: ZRP 2010, 46 (47).

28 Diese und die folgenden statistischen Angaben wurden den jährlichen Pressemitteilungen des BMI zur PMK entnommen.

29 Vgl. Pressemitteilung des BMI vom 30.03.2007; vgl. auch Reichard (Anm. 17), S. 17.

30 Vgl. Steinhaus: Einführung zum polizeilichen Definitionssystem „politisch motivierte Kriminalität (PMK) “, in: Ministerium des Inneren des Landes Brandenburg (Hg.): Info 110 2/2003, S. 18; Ziercke: Lagebild extremistischer Gewalt in Deutschland, in: Egg (Hg.): Extremistische Kriminalität. Kriminologie und Praxis, 2006, S. 61 (66).

31 Vgl. Liebich-Frels: Probleme der Definition und Erfassung fremdenfeindlicher Straftaten, in: DPolBl 2005, S. 8 (10); Innenministerium Nordrhein-Westfalen: Verfassungsschutzbericht NRW 2001, S. 185.

32 Vgl. BMI/BMJ (Anm. 24), S. 136

33 Vgl. Jacobs/Potter: Hate Crimes - criminal law and identity politics, 1998, S. 22

$34 \mathrm{Vgl}$. BMI/BMJ (Anm. 4), S. 269.

35 Vgl. BMI/BMJ (Anm. 4), S. 270.

$36 \mathrm{Vgl}$. BMI/BMJ (Anm. 24), S. 134

37 Vgl. Ziercke (Anm. 30), S. 66 m.w.N

38 Vgl. Singer (Anm. 18), 32 (36).

39 Vgl. Singer (Anm. 18), 32 (35).

40 Vgl. Kubink (Anm. 3), 325 (331).

41 Vgl. Singer (Anm. 18), 32 (35).

42 Vgl. Singer (Anm. 18), 32 (36).

43 Vgl. Willems (Anm. 9), S. $16 \mathrm{f}$.

44 Vgl. Kaiser: Kriminologie, 3. Aufl. 1996, $\mathbb{S} 36$ Rn. 49.

45 Vgl. Sutterlüty: Gewaltkarrieren. Jugendliche im Kreislauf von Gewalt und Missachtung, 2002, S. 313; Koblstruck: "Hate Crimes" - Anmerkungen zu einer aktuellen Diskussion, in: Berliner Forum Gewaltprävention Nr. 16, Dokumentation des 4. Berliner Präventionstages am 13.11.2003, 2004, S. 64 (70 f.).

46 Vgl. Graser/Fittkau: Die Gewalt ist rechts, in: Kriminalistik 2008, 32 (39).

47 Näher hierzu Baldus: Hate Crime. Gesetze zur effektiven Bekämpfung von Rechtsextremismus und Fremdenfeind-lichkeit in Deutschland?, 2003, S. 8 ff. u. 21; Coester: Hate Crimes. Das Konzept der Hate Crimes aus den USA unter besonderer Berücksichtigung des Rechtsextremismus in Deutschland, 2008, S. $52 \mathrm{ff}$.

48 Vgl. Koblstruck (Anm. 45), S. 64 (67); ausführlich zum Konzept der "hate crimes“ in den USA Coester (Anm. 47), S. $19 \mathrm{ff}$

49 Vgl. Kubink (Anm. 3), S. 337.

50 Vgl. Kohlstruck (Anm. 45), S. 64 (69).
51 Vgl. Kohlstruck (Anm. 45), S. 64 (69).

52 Anders aber: Schneider (Anm. 14), 21 (22 ff.).

53 Vgl. Kohlstruck (Anm. 45), S. 64 (69).

54 Vgl. Walter: Jugendkriminalität, 3. Aufl. 2005 , Rn. 129 b.

$55 \mathrm{Vgl}$. Bannenberg/Rössner/Coester: Hasskriminalität, extremistische Kriminalität, politisch motivierte Kriminalität und ihre Prävention, in Egg (Hg.): extremistische Kriminalität. Kriminologie und Praxis, 2006, S. 17.

56 Vgl. Walter (Anm. 54), Rn. 209.

57 Vgl. Albrecht/Lamnek: Jugendkriminalität im Zerrbild der Statistik, 1979; S. 13; Walter (Anm. 54), Rn. 210.

58 Vgl. Jacobs/Potter (Anm. 33), S. 22

59 Vgl. Kubink (Anm. 5), 308 (312).

$60 \mathrm{Vgl}$. Giminéz-Salinas: Hasskriminalität und Prävention, in: BMJ (Hg.): Tagungsband $\mathrm{Ar}-$ beitsgruppe „Primäre Prävention von Gewalt gegen Gruppenangehörige, 2003, S. 85 (92).

$61 \mathrm{Vgl}$. Kohlstruck (Anm. 45), S. 64 (71).

62 Vgl. Kohlstruck (Anm. 45), S. 64 (71).

63 Vgl. Schneider: Opfer von Hassverbrechen junger Menschen: Wirkungen und Konsequenzen, in: MSchrKrim 2001, 357 (363 ff.).

64 So Koblstruck (Anm. 45), S. 64 (71).

65 Vgl. Kohlstruck (Anm. 45), S. 64 (71); Baldus (Anm. 47), S. 19

66 Vgl. Kubink (Anm. 5), 308 (311).

67 So Schneider (Anm. 14), 21 (22).

68 Vgl. Kubink (Anm. 5), 308 (311).

69 So Schneider (Anm. 14), 21 (24).

70 Vgl. Kubink (Anm. 3), 325 (339).

71 Vgl.; Pfahl-Traughber: Ursachen rechtsextremistisch motivierter Gewalt, in: Kriminalistik 2004, S. 38 (39 ff.); Schneider: Kriminologie der Hassdelikte, in: BewHi 2003, 115 (123 f.) Bannenberg/Rössner/Coester (Anm. 55), S. $17 \mathrm{ff}$.

72 Vgl. Neubacher (Anm. 2), S. 23; Coester (Anm. 47), S. 375.

73 Selbst wenn den statistischen Daten eine hinreichende Aussagekraft zukäme, spräche ob der kontinuierlich zurückgehenden Fallzahlen (s.o. III. 3.) nichts für derartige Vorschläge. Eingehend zur Problematik entsprechender Gesetzesänderungen Keiser (Anm. 27), 46 (47 ff.); vgl. aber auch Reichard (Anm. 17), S. 393 f., der aus Klarstellungsgesichtspunkten dafür plädiert, den Strafzumessungskatalog des $\$ 46$ II StGB um fremdenfeindliche Motive zu erweitern. 